\title{
ON THE PHOTOMETRIC BEHAVIOUR OF THE CENTRAL STAR OF THE PLANETARY NEBULA Sh2-71
}

\author{
J. JURCSIK \\ Konkoly Observatory of the Hungarian Academy of Sciences
}

The central star of Sh2-71 was observed with the $1 \mathrm{~m}$ telescope using UBV $(\mathrm{RI})_{C}$ photometric system at Konkoly Observatory in the seasons of 1990-92. A period of 68.06 was found with variable amplitude and/or lightcurve shape. The amplitude is high, close to 1 magnitude. On the basis of old photographic observations (Kohoutek 1979) this period has not changed during the century. No significant colour variation was detected, but the nebular contamination should distort the colour curves. According to the five colour data the central object is a highly reddened B5 dwarf about 1700 pc distant. The nebular properties, however, indicate an evolved, higher mass, lower luminosity exciting source, therefore it is plausible to regard the central object as a binary. The light variation might be caused by obscuration of the B star by outflowing matter from the true nucleus. Bipolar outflow of 3" dimension has been detected on high dispersion $\mathrm{H} \alpha$ spectra of the central star (Sabbadin et al. 1985; Cuesta et al. 1990). Further spectroscopic observations are highly needed in order to find the exact description of the system.

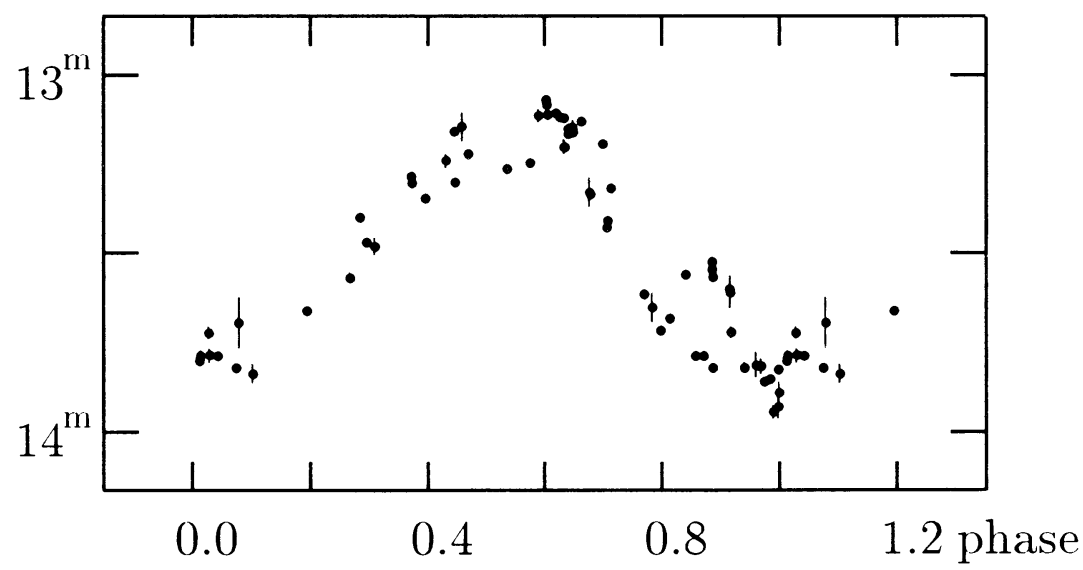

Fig. 1. The folded V lightcurve of the B star in the centre of Sh2-71 with the ephemeris Tmin=JDH2448190.0+68d.064E. The scatter of the curve is larger than the observational error indicating real changes in the light variation.

\section{References}

Cuesta, L., Phillips, J. P., Mampaso, A.: 1990, Ap. Space. Sci. 171, 163.

Kohoutek, L.: 1979, IBVS 1672.

Sabbadin, F., Ortolani, S., Bianchini, A.: 1985, Monthly Not. Roy. Astron. Soc. 213, 563. 